\section{UNANOVA: An unweighted-means analysis of variance program for the TI-59 programmable calculator}

\author{
NEIL R. SHAPIRO and BRUCE C. DUDEK \\ State University of New York, Albany, New York 12222
}

A recent article demonstrated that the usefulness of the Texas Instruments (TI) advanced programmable calculators is not restricted to data analysis, but rather, it extends to data acquisition and control of experimental events (Robinson, 1979). Despite the flexibility and power of these calculators, and although a variety of statistical programs are available via the TI solid state program modules, the flexibility of software to perform analysis of variance (ANOVA) is severely limited. The program described here permits the calculator to be used to conduct an ANOVA on completely randomized factorial designs, with equal or unequal group sizes. The program was written for the TI 59 calculator, and it will print the results. ${ }^{1}$

Program Description. The program performs an unweighted-means analysis of variance (UNANOVA) on any completely randomized single- or multiplefactor design (nonhierarchical). The number of crossed variables is limited in the maximum number of groups that may be entered. Specifically, this maximum is defined by the equation,

$$
\mathrm{k}=52-\mathrm{N} \text {, }
$$

where $\mathrm{k}$ is the maximum number of groups and $\mathrm{N}$ is the maximum group size to be entered. The maximum number of subjects in any group for a particular design can be ascertained by setting $k$ equal to the number of groups to be entered and solving Equation 1 for $\mathrm{N}$.

The program allows data to be stored or entered via magnetic cards, permitting transformations to be performed without reentry of individual data points. When group data are stored on cards, the group mean is concurrently stored, simplifying transformations involving group means (e.g., an analysis of absolute deviation scores).

To use the program, one simply enters individual data points (or an entire group via a card). Pressing a user-defined key indicates that the group's data are now in memory. The calculator then displays or prints the group size and mean. The user may at this point delete the group or enter it into the ANOVA. In the latter case, a standard deviation is displayed, followed by the total number of groups that have been entered to that point. After the last group is entered, pressing a second userdefined key computes and displays the total number of groups $(\mathrm{k})$, the harmonic mean of group size $(\mathrm{N})$, the between- and within-cell sum of squares (SS), the associated degrees of freedom, the MS for each, and the omnibus $F$ value. The calculator then initiates a routine for comparisons between means (see Keppel, 1973), in which the user enters weighting coefficients in response to prompting (the calculator displays each group mean successively). This "decomposition" routine can be used to break down the between-cell SS into variance due to individual factors in the factorial design, make planned or post hoc comparisons between means, perform a trend analysis, or perform an analysis of simple main effects. For example, to break down the between-cell SS for a 2 by 2 factorial design, one assigns a weight of -1 to both cells at one level of the first variable and a +1 to both cells at the other level. The calculator then displays the MS for that comparison and the $F$ value using the within-cell MS in the denominator. Variance attributable to the second factor is similarly derived. The interaction of the two variables is derived by entering a set of coefficients representing the product of the sets used for each of the first two comparisons. A users' manual for the program describes this procedure in more detail and includes examples of both simple and complex factorial designs. While the program is not designed to directly derive analyses of designs that are not complete between-group factorials (e.g., fractional factorials, hierarchical or nested designs, or repeated measures), a working knowledge of these designs should permit the user to utilize UNANOVA to vastly reduce any manual computations involved in such analyses. This procedure involves successive analyses that are then pieced together.

Program Availability. The users' manual and complete program listing can be obtained from the second author, Department of Psychology, State University of New York at Albany, 1400 Washington Avenue, Albany, New York 12222. A $\$ 2$ fee to cover copying and mailing costs would be appreciated.

\section{REFERENCES}

KePPEL, G. Design and analysis: A researcher's handbook Englewood Cliffs, N.J: Prentice-Hall, 1973.

RoBinson, G. H. Programming experiments with pocket programmable calculators. Behavior Research Methods \& Instrumentation, 1979, 11, 61-63.

\section{NOTE}

1. The program can be easily adapted for use with other programmable calculators, including the TI-58. However, without some form of program storage capability, the usefulness of such a program must be weighted against the need to enter the $393-$ step routine each time the calculator is turned on.

(Accepted for publication October 16, 1980.) 